\title{
UNIQUE CONTINUATION THEOREMS IN GAUGE THEORIES
}

\author{
CLIFFORD HENRY TAUBES
}

\begin{abstract}
Gauge theoretic version of Aronszajn's unique continuation principle are proved here for the anti-self dual equations. (This system of non-linear, partial differential equations does not have an elliptic symbol.) For example: Two anti-self dual connections on a 4-manifold which are gauge equivalent to infinite order at a single point are gauge equivalent on some neighborhood of any point.
\end{abstract}

The purpose of this note is to state and prove five unique continuation theorems for solutions of the anti-self dual equations on 4-dimensional Riemannian manifolds. These theorems make global statements about pairs of solutions to the anti-self dual equations which happen to agree on some subspace. The first theorem is an analog to Aronszajn's unique continuation principle [Ar] for solutions to Laplace's equation on a manifold. The second theorem is an analog which considers anti-self dual connections on manifolds with boundary which agree on the boundary.

The second theorem generalizes a result of Donaldson [D] about anti-self dual connections on holomorphic bundles over Kahler manifolds with nonempty boundary. Neither theorem can be obtained (to the author's knowledge) by simple quoting theorems about elliptic equations (e.g. [Ar]), for the anti-self dual equations do not linearize as elliptic equations for the connection.

The third theorem is an infinitesimal version of the second, and the fourth and fifth theorems are essentially corollaries to the first and second; they concern anti-self dual connections on a 4-dimensional manifold which are reducible on some subset. These last two theorems generalize Lemma 4.3.2 in [DK].

Supported in part by the NSF. 


\section{The Theorems}

The setting for the first theorem is as follows: Consider a smooth, connected, 4-dimensional Riemannian manifold $W$. Let $G$ be a compact, simple Lie group, and let $P \rightarrow M$ be a principal $G$ bundle. A connection $A$ on $P$ will be called anti-self dual if $A$ 's curvature 2-form, $F_{A}$, obeys $F_{A}+* F_{A}=0$. (See, e.g. [AHS].)

A pair of connections on $P, A$ and $A^{\prime}$, are said to be gauge equivalent if there is an automorphism of $P$ which pulls $A^{\prime}$ back to equal $A$. Say that $A$ and $A^{\prime}$ are locally gauge equivalent if each point in $W$ has a neighborhood on which $A$ and $A^{\prime}$ restrict to be gauge equivalent. (These two notions are not generally equivalent, as any two connections with vanishing curvature will be locally gauge equivalent to each other.) Say that $A$ and $A^{\prime}$ are gauge equivalent to infinite order at a point, $p$, of $W$ if the following is true: There is a neighborhood, $U \subset W$, of $p$ and an automorphism, $\eta$, of $\left.P\right|_{U}$, such that $\eta^{*} A^{\prime}-A$ vanishes at $p$ as well as all partial derivatives of $\eta^{*} A^{\prime}-A$ to all orders. (Even though $\eta^{*} A^{\prime}-A$ is a section of a vector bundle over $W$, the assertion of the vanishing of $\eta^{*} A^{\prime}-A$ and its partial derivatives up to some order at a point is well defined and independent of choices of coordinates, and bundle trivializations.)

Theorem 1. Let $W$ be a smooth, oriented 4-manifold with Riemannian metric. Let $G$ be a compact Lie group. Consider a principal $G$ bundle $P \rightarrow W$. Let $A$ and $A^{\prime}$ be anti-self dual connections on $P$ which are gauge equivalent to infinite order at some point $p$ in $W$. Then $A$ and $A^{\prime}$ are locally gauge equivalent.

Note that the conclusions of Theorem 1 cannot, in general, be strengthened to claim that $A$ and $A^{\prime}$ are globally gauge equivalent. Just consider the flat connections. (Theorem 1 is stated as part of Theorem 1.5 in unpublished versions of $[\mathrm{T}]$, but the proof in these versions of $[\mathrm{T}]$ has a gap. The published version refers here.)

Here is the setting for the second theorem: Consider a smooth, connected, 4-dimensional, Riemannian manifold $W$. Assume that $W$ has a smooth, compact, non-empty boundary, $\partial W$. Let $M \subset \partial W$ be a component, and let 
$i: M \rightarrow W$ denote the boundary inclusion. Again, let $P \rightarrow W$ be a principal bundle with structure group $G$, a compact Lie group.

The second theorem asserts that the boundary values of an anti-self dual connection essentially determine the connection.

Theorem 2. Let $W, M$ and $P$ be as described above. Let $A$ and $A^{\prime}$ be antiself dual connections on $P$ which are Sobolev class $L_{3}^{2}$ on some neighborhood of the boundary. If $i^{*} A$ is gauge equivalent to $i^{*} A^{\prime}$ on $M$, then $A$ is locally gauge equivalent to $A^{\prime}$ on $W$.

(A connection $A$ on a bundle $Q$ over a compact manifold with (or without) boundary, $X$, is of Sobolev class $L_{k}^{2}$ for integer $k \geq 0$ when the following conditions are met: Let $B \subset X$ be any open subset with compact closure such that $\left.Q\right|_{B}$ is trivial. For any smooth trivialization $\eta: B \times\left. G \rightarrow Q\right|_{B}$, the components of $\eta^{*} A$ and their partial derivatives up to $k$-th order should be square integrable over $B$. After embedding $G$ faithfully in a matrix group, a similar definition describes the Sobolev class $L_{k}^{2}$ automorphisms of $Q$. One can also define these Sobolev classes for fractional $k$, see [Ad].)

(An anti-self dual connection which is of Sobolev class $L_{1}^{2}$ on $X$ will be smooth on the interior of $X$; see [U]. If the connection is of Sobolev class $L_{3}^{2}$, then it will be continuous (at least) as a 1 -form on $Q$.)

As remarked, Donaldson [D] proved a related theorem about anti-self dual connections on holomorphic bundles over Kahler manifolds with boundary.

Theorem 2 also has an infinitesimal version which is stated in Theorem 3 , below. However, a two-part digression is required to first set the stage: Let $W$, $M$ and $P$ be as described in Theorem 2. To start Part 1 of the digression, fix a point $p \in M$ and let $G^{0}$ denote the group of Sobolev class $L_{4}^{2}$ automorphisms of $P$ which restrict to the identity at $p$. Let $\mathfrak{M}^{0}$ denote the quotient by $G^{0}$ of the space $\mathfrak{m}$ of Sobolev class $L_{3}^{2}$, anti-self dual connections on $P$. It is proved in Proposition 2.2 of [T] that $\mathfrak{M}^{0}$ is a smooth manifold, a priori of infinite dimensions. With $\mathfrak{M}^{0}$ understood, introduce $B^{0}(M)$ to denote the quotient of the space of Sobolev class $L_{5 / 2}^{2}$ automorphisms of $P$ which restrict to the identity over $P$. This $B^{0}(M)$ is a smooth, infinite dimensional manifold. End Part 1 of the digression. 
Part 2 of the digression introduces the notion of a locally reducible connection on $P$. Here, a connection $A$ will be said to be locally reducible if every point in $W$ has a neighborhood on which there exists a non-zero, $A$-covariantly constant section of $P \times_{G} g$.

Theorem 3. Let $W, M$ and $P$ be as in Theorem 2. Then, the pull-back by the boundary inclusion map $i: M \rightarrow W$ defines a smooth $G$-equivariant map $\iota: \mathfrak{M}^{0} \rightarrow B^{0}(M)$ which is an immersion at orbits of connections which are not locally reducible on $W$. (Here, the term "immersion" is used to described the map whose differential is everywhere injective.)

Theorems 2 and 3 have some relevance vis-a-vis Floer cohomology, as will now be explained. When defined, Floer cohomology ([F] and [TWZ]) for a 3manifold $M$ is constructed from the moduli space of anti-self dual connections on $\mathbb{R} \times M$. There is a formal analogy between Floer cohomology and the cohomology of a compact manifold as computed using Morse theory. (See, e.g. [CJS].) The analogy starts with the observation that the anti-self dual equations on $W=$ interval $\times M$ are, formally, the gradient flow equations for a functional on the space of equivalence classes of connections on a principal bundle over the 3-manifold in question.

A gradient flow (with time parameter $t \in[0,1)$ ) of a function $f$ on a finite dimensional, Riemannian manifold defines a map from $[0,1)$ into the manifold in question. And, as the solution of a first order ODE for finitely many unknowns, this map is determined completely by its initial conditions at $t=0$.

Formally, the anti-self dual equations on $[0,1) \times M$ can be thought of as a first order ODE in infinitely many unknowns, but the standard theorems about the initial values determining the flow do not hold. None-the-less, because of Theorem 2, one knows that the time zero values of anti-self dual connections on $[0,1) \times M$ essentially determine the connection at all later times.

By the way, for a gradient flow on a compact, finite dimensional manifold, every point is the starting point of some flow line defined on $[0,1)$. There is no direct analogy for anti-self dual connections on $[0,1) \times M$. Indeed, one can write down orbits of smooth connections in $B^{0}(M)$ which cannot be extended as anti-self dual connections on $[0,1) \times M$ or on $(-1,0] \times M$. (As an exercise, 
try to do so with a $U(1)$ connection on a trivial $U(1)$ bundle over $T^{3}$ or $S^{3}$.) However, it is reasonable to conjecture that the initial values of anti-self dual connections on $[0,1) \times M$ are dense in $B^{0}(M)$. There is some evidence for this assertion: Look at the restriction map to $B^{0}(M)$ from the space $\mathfrak{M}^{0}$ as defined on $[0,1) \times M$. Then, at any point in $\mathfrak{M}^{0}$, the closure of the image of the differential of this map has empty compliment (consider the proof of Proposition 2.2 in [T].) See also Marini [M].

The last two theorems generalize Lemma 4.3.2 in [DK] which asserts that an anti-self dual connection which is reducible on a ball in $W$ is locally reducible on $W$. The context for Theorem 4, below, is the same as for Theorem 1. Theorem 4 discusses connections which are reducible to infinite order at a point in $W$. (A connection $A$ on $P$ is reducible to infinite order at a point $p \in W$ if there exists a section of $P \times_{G} P$ which is not zero at $p$, but whose $A$-covariant derivative vanishes to infinite order at $p$.)

Theorem 4. Let $W$ and $P$ be as described in Theorem 1. Let $A$ be an antiself dual connection on $P$ which is reducible to infinite order at some point in $W$. Then $A$ is locally reducible on $W$.

The argument for Theorem 4 is similar to that for Lemma 4.3.2 in [DK].

The statement of Theorem 5, below, requires a preliminary digression to introduce the following terminology: Let $G$ be a compact, simple Lie group, and introduce $\underline{G} \equiv G / \operatorname{Center}(G)$. Introduce $\rho: G \rightarrow \underline{G}$ to denote the natural group homomorphism, and let $A d \rho$ denote the composition of $\rho$ with the adjoint action of $\underline{G}$ on itself.

Now, say that a connection $A$ on a principal $G$ bundle, $P$, over a manifold is $\underline{G}$-reducible if there is a non-trivial, $A$-covariantly constant section of the associated bundle $P \times_{A d \rho} \underline{G}$. (If $A$ is of Sobolev class $L_{k}^{2}$, then the section in question will be required to be Sobolev class $L_{k+1}^{2}$.) Say that $A$ is locally $\underline{G}$-reducible if every point has a neighborhood to which $A$ restricts as a $\underline{G}-$ reducible connection.

Theorem 5. Let $W, M$ and $P$ be as described in Theorem 2. Let $A$ be a Sobolev class $L_{3}^{2}$, anti-self dual connection on $P$. Suppose that $i^{*} A$ is $\underline{G}_{-}$ reducible on $M$. Then $A$ is locally $\underline{G}$-reducible on $W$. 
The remainder of this note contains the proofs of these theorems.

\section{Proof of Theorem 1}

The purpose of this section is to present a proof of Theorem 1. Before starting, remark that the strategy here will follow closely that of [Ar]. Roughly, this strategy argues as follows: Given $A$ and $A^{\prime}$, introduce the set $U \subset W$ of points which have open neighborhoods to which $A$ and $A^{\prime}$ restrict as gauge equivalent connections. By definition, $U$ is open. If $U$ is also closed and non-empty, then $U=W$. To prove that $U$ is non-empty, one must establish Theorem 1 in the case where $W \subset \mathbb{R}^{4}$ is an open 4-ball about the origin, and where $A$ and $A^{\prime}$ are anti-self dual connections on $\mathbb{R}^{4} \times G$ which are gauge equivalent to infinite order at the origin. Here, the metric on $W$ need not be Euclidean.

Note: Proving this special case also proves that $U$ is closed. Here is why: The connections $A$ and $A^{\prime}$ will be gauge equivalent to infinite order at every point in $U$ and so they will be locally gauge equivalent at every point in the closure of $U$. Then, to show that a point in the closure of $U$ has a neighborhood on which $A$ and $A^{\prime}$ are gauge equivalent, one need only appeal to the special case above. Thus, Theorem 1 is proved in general if it can be established for the case of a ball in $\mathbb{R}^{4}$ as described above.

With the preceding understood, let $d s^{2}$ be a smooth metric on an open set in $\mathbb{R}^{4}$ which contains the origin. Use gaussian normal coordinates to write $d s^{2}$ on a ball, $B$, about the origin, as

$$
d s^{2}=d x_{\alpha} \otimes d x_{\alpha}+h,
$$

where $h$ obeys the conditions

(1) $|h|(x) \leq c \cdot|x|^{2}$.

(2) $|\nabla h|(x) \leq c \cdot|x|$.

(3) The unit radial vector field $\partial_{r}$ on $\mathbb{R}^{4}-0$ is tangent to the $d s^{2}$-geodesic through 0 .

Now, let $G$ be a compact, simple Lie group. Let $A$ and $A^{\prime}$ be a pair of connections on $B \times G$ which are anti-self dual for the metric $d s^{2}$. Let $\Gamma$ denote the product connection on $B \times G$. Write $A=\Gamma+a$ with $a$ being 
a section over $B$ of $T^{*} B \times \mathfrak{g}$. Thus, the anti-self dual equations say that $P_{+} F_{A}=P_{+}(d a+a \wedge a)=0$. (Here, $P_{+}: \wedge^{2} T^{*} B \rightarrow \wedge^{2} T^{*} B$ is the $\left(d s^{2}\right)$ orthogonal projection onto the subbundle of self dual (with respect to $d s^{2}$ ) 2-forms.)

Likewise, write $A^{\prime}=\Gamma+a^{\prime}$.

suppose that $a$ and $a^{\prime}$ agree to infinite order at the origin. Here is the goal: find $s: B \rightarrow G$ such that $a^{\prime \prime} \equiv s \cdot a \cdot a^{-1}+s \cdot d s^{-1}$ and $a$ agree on a non-empty open set about the origin. With the preceding understood, the following steps produce the gauge transformation $s$ as above.

Step 1. Find separate gauge transformations of $A$ and $A^{\prime}$ (if necessary) so that after applying these gauge transformations,

(1) $\left.a\right|_{0}=\left.a^{\prime}\right|_{0}=0$

(2) The contractions of $a$ and $a^{\prime}$ with $\partial_{r}$ satisfy $\partial_{r} \iota a=\partial_{r} \iota a^{\prime}=0$.

The "gauge" change which affects (1.3) for $A$ is obtained by parallel transport from 0 along radial geodesics. Thus, the invocation of (1.3) does not affect the assumption that $a$ and $a^{\prime}$ agree to infinite order at 0 .

Step 2. Let $u \equiv a-a^{\prime}$. then $u$ satisfies $u(0)=0, \partial_{r} \iota u=0$ and obeys the partial differential equation

$$
P_{+} d u+\frac{1}{2} \cdot P_{+}\left(u \wedge\left(a+a^{\prime}\right)+\left(a+a^{\prime}\right) \wedge u\right)=0 .
$$

Notice that (1.3) and (1.4) imply that

$$
\left|P_{+} d u\right|^{2} \leq c_{1} \cdot|x|^{2} \cdot|u|^{2}
$$

for some constant $c_{1}$ which is determined by the $C^{1}$-norms of $a$ and $a^{\prime}$.

Step 3. The strategy now will be to mimic, as much as is possible, the approach used by Aronszajn in [Ar]. The following lemma-which is proved below in Step 6 is crucial.

Lemma 1.1. There is a constant $c>0$, and there exists an increasing, smooth function $\rho:[0,1] \rightarrow[0,1]$; and these have the following properties:

(1) They are determined by the metric $d s^{2}$ on $B$.

(2) $\rho^{-1}(0)=0$ and $\left(\partial_{t} \rho\right)(0)>0$. 
(3) Let $r \in(0,1)$ be less than the diameter of $B$, and let $B_{r} \subset B$ denote the ball of radius $r$ about the origin. Let $v$ be a smooth, $\mathfrak{g}$-valued 1-form on $B$ with compact support in $B-\{0\}$ and with $\partial_{r} \iota v=0$. Then for all $\alpha>0$,

$$
c \cdot r^{2} \int_{B_{r}} \rho(|x|)^{-2 \alpha}\left|P_{+} d v\right|^{2} \geq \int_{B_{r}} \rho(|x|)^{-2 \alpha} \cdot|v|^{2} .
$$

Step 4. Fix a smooth, non-negative function $\beta$ on $[0,1]$ which vanishes on $[3 / 4,1]$ and which is identical to 1 on $[0,1 / 4]$. Then, fix $r_{0}>0$ so that $B_{r_{0}} \subset B$. For $r<r_{0}$, define $\beta_{r}: B \rightarrow[0,1]$ to have value at $x$ equal to $\beta(|x| / r)$.

Argue as in [Ar] that $v \equiv \beta_{r} \cdot u$ obeys (1.6) for all $\alpha>0$. (Because $u$ vanishes to all orders at the origin, the integrals in (1.6) are absolutely convergent. With this understood, it is relatively straightforward to approximate $\beta_{r} \cdot u$ by a sequence, $\left\{u_{n}\right\}$, where each $u_{n}$ has compact support in $B-0$ and where the sequences of values of both sides of (1.6) for $\left\{v=u_{n}\right\}$ converge to the respective values of both sides of (1.6) for $v=\beta_{r} \cdot u$.)

Step 5. Use (1.5) with (1.6) to conclude that

$$
\int_{B_{r}-B_{r / 2}} \rho(|x|)^{-2 \alpha} \cdot|u|^{2} \geq \int_{B_{r / 2}} \rho(|x|)^{-2 \alpha} \cdot|u|^{2},
$$

for all $\alpha>0$ if $r$ is sufficiently small. Then, note how (1.7) implies that

$$
\int_{B_{r}-B_{r / 2}}\left(\frac{\rho\left(\frac{r}{2}\right)}{\rho(|x|)}\right)^{2 \alpha} \cdot|u|^{2} \geq \int_{B_{r / 2}}|u|^{2},
$$

for all $\alpha$. Since $\rho$ is increasing on [0,1], Equation (1.8) can hold for all $\alpha$ only if $u \equiv 0$ on $B_{r / 2}$.

Step 6. This last step proves Lemma 1.1. The proof of this lemma requires several parts. The first part defines the function $\rho$. The definition requires the following digression: Think of $S^{3}$ as the unit sphere in $\mathbb{R}^{4}$. Let $T_{0} \equiv-\ln \left(r_{0}\right)$ and introduce the diffeomorphism

$$
\Psi:\left[T_{0}, \infty\right) \times S^{3} \rightarrow B_{r_{0}}-0
$$

which sends $(t, y)$ to the point $e^{-t} \cdot y \in \mathbb{R}^{4}$. Pull $d s^{2}$ back by $\Psi$ to obtain a metric $e^{-2 t} \cdot\left(d t^{2}+m\right)$, where $\left.m\right|_{t} \equiv m_{t}$ is a smooth metric on $S^{3}$. 
The set of metrics $\left\{m_{t}\right\}_{t>T_{0}}$ defines a smooth map, which will also be denoted by $m$, from $\left[T_{0}, \infty\right)$ into the Frechet space of smooth metrics on $S^{3}$. The limit as $t \rightarrow \infty$ of $\left\{m_{t}\right\}$ converges as $t \rightarrow \infty$ to the standard round metric on $S^{3}$. This convergence is exponentially fast. In particular,

(1) $\left|m_{t}-m_{\infty}\right|_{m} \leq c_{2} \cdot e^{-2 t}$

(2) $\left|\partial_{t} m_{t}\right|_{m} \leq c_{2} \cdot e^{-2 t}$

where $\partial_{t}$ denotes the derivative with respect to the coordinate $t \in\left[T_{0}, \infty\right)$ and where $c_{2}$ is some constant.

End the digression.

Following an idea from [Ar], define $\rho$ by

$$
\rho(s)=\exp \left[-\int_{0}^{-\ln (s)} d \tau \cdot \exp \left(1000 \cdot c_{2} \cdot e^{-2 t}\right)\right]
$$

where the $c_{2}$ is the constant in (1.10).

Part 2 of the proof of Lemma 1.1 involves translating (1.6) to an inequality for $\mathfrak{g}$-values 1 -form on $[T, \infty) \times S^{3}$ with $T>T_{0}$. Indeed, (1.6) is implied by an analogous inequality for $\nu \equiv \Psi^{*} v$ on $[T, \infty) \times S^{3}$. In deriving the inequality for $\nu$, remember that the operator $P_{+} d$ pulls back to $[T, \infty) \times S^{3}$ as $P_{-} d$ (the operator $d$ and the subbundles $P_{ \pm} \bigwedge^{2} T^{*}$ pull-back naturally under conformal diffeomorphisms, but $\Psi$ reverses orientation).

Then (1.6) is equivalent to the following assertion:

Lemma 1.1'. There exists $c>0$ with the following significance: Let $T>T_{0}$ be given. Let $\nu$ be a compactly supported, $\mathfrak{g}$-valued 1 -form on $[T, \infty) \times S^{3}$, and suppose that the contraction $\partial_{t} \iota \nu$ vanishes so $\nu$ has no dt component. Let $\underline{\rho}(t) \equiv \rho\left(e^{-t}\right)$, with $\rho$ as in (1.11). Then, for all $\alpha>0$,

$$
c \cdot e^{-2 t} \int_{[T, \infty) \times S^{3}} \underline{\rho}(t)^{2 \alpha} \cdot\left|P_{-} d \nu\right|_{g}^{2} \cdot d \operatorname{vol}_{g} \geq \int_{[T, \infty) \times S^{3}} e^{-2 t} \underline{\rho}(t)^{2 \alpha} \cdot|\nu|_{g}^{2} \cdot d \operatorname{vol}_{g} \cdot
$$

Here, $P_{-}$is the anti-self dual projection for the metric $g \equiv d t^{2}+m$, and $|\cdot|_{g}$ is the norm for said metric, while $d \mathrm{vol}_{g}$ is $g$ 's volume form on $[T, \infty) \times S^{3}$.

Thus Lemma 1.1 is proved with a proof of Lemma $1.1^{\prime}$. 
Proof of Lemma 1.1'. The proof begins by analysing the integral on the left side of (1.12). Introduce $\underline{d}$ to denote the exterior derivative along the constant $t$ hypersurfaces. (So the contraction of $\underline{d} f$ with $\partial_{t}$ is zero.) Since $\nu$ has no $d t$ component, one can think of $\nu$ in (1.12) as a time dependent section of $T^{*} S^{3} \otimes \mathfrak{g}$. Let $\partial_{t} \nu$ denote its derivative in the $t$-direction. Then,

$$
\left|P_{-} d \nu\right|_{g}^{2}=\left|\partial_{t} \nu-* \underline{d} \nu\right|_{m}^{2}
$$

where $|\cdot|_{m}$ is the norm on $T^{*} S^{3}$ using the metric $m$; and where $*$ is the Hodge star on $S^{3}$ for the metric $m$.

Next, one should introduce $w \equiv \underline{\rho}^{\alpha} \cdot \nu$ and note that (1.12) can be rewritten in terms of $w$ as

$$
c \cdot e^{-2 t} \int_{[T, \infty) \times S^{3}}\left|\partial_{t} w+\alpha \cdot f \cdot w-* \underline{d} w\right|_{m}^{2} \cdot d \operatorname{vol}_{g} \geq \int_{[T, \infty) \times S^{3}} e^{-2 t}|w|_{m}^{2} \cdot d \operatorname{vol}_{g}
$$

where

$$
f(t) \equiv-\underline{\rho}^{-1} \cdot \partial_{t} \underline{\rho}=\exp \left(1000 \cdot c_{2} \cdot e^{-2 t}\right)
$$

The integral on the left side of (1.14) can be rewritten as

$$
\int_{[T, \infty) \times S^{3}}\left(\left|\partial_{t} w\right|_{m}^{2}+|\alpha \cdot f \cdot w-* \underline{d} w|_{m}^{2}+2 \cdot\left\langle\partial_{t} w, \alpha \cdot f \cdot w-* \underline{d} w\right\rangle_{m}\right) d t \cdot d \operatorname{vol}_{m}
$$

where $\langle,\rangle_{m}$ is the inner product on $T^{*} S^{3}$ using the metric $m$.

It turns out that all three terms in the integrand of (1.16) integrate to non-negative numbers. Indeed, the first two terms in (1.16) are obviously non-negative. And, integration by parts can be used to analyze the integral of the last term in the integrand of (1.16). In particular, as $w$ has compact support on $[T, \infty) \times S^{3}$, the last term in (1.16) is equal to

$$
\alpha \cdot \int_{[T, \infty) \times S^{3}}\left(-\partial_{t} f \cdot|w|_{m}^{2}-f \cdot\langle w, H \cdot w\rangle_{m}\right) d t \cdot d \operatorname{vol}_{m}
$$

where $\left.H\right|_{t}$ is an endomorphism of $T^{*} S^{3}$ which is constructed out of $\partial_{t} m$. (Integration by parts shows that the term $\left\langle\partial_{t} w, * \underline{d} w\right\rangle_{m}$ contributes zero to (1.16). In fact, (1.17) arises when integrating $\left\langle\partial_{t} w, \alpha \cdot f \cdot w\right\rangle_{m}$ by parts; the 
$H$-term contains all time derivatives of the metric $m$ and its volume 3 -form.) In any event,

$$
\left|\langle w, H \cdot w\rangle_{m}\right| \leq 10^{2} \cdot\left|\partial_{t} m\right|_{m} \cdot|w|_{m}^{2} \leq 10^{2} \cdot z \cdot e^{-2 t} \cdot|w|_{m}^{2}
$$

Because $-\partial_{t} f=2 \cdot 10^{3} \cdot z \cdot e^{-2 t} \cdot f$, the integral in (1.17) (which equals the integral of the last term in the integrand in (1.16)) is positive as claimed.

Thus, the integral in (1.16), and so the integral on the left side of (1.12), is greater than

$$
\int_{[T, \infty) \times S^{3}}\left|\partial_{t} w\right|_{m}^{2} d t \cdot d \operatorname{vol}_{m}
$$

The proof of Lemma 1.1' continues by analyzing the integral on the right side of (1.12). Set $w \equiv \underline{\rho}^{\alpha} \cdot \nu$ and this integral becomes

$$
\int_{[T, \infty) \times S^{3}} e^{-2 t} \cdot|w|_{m}^{2} d t \cdot d \operatorname{vol}_{m} .
$$

Since the metric $m$ on $[T, \infty)$ is uniformly close to the metric $m_{\infty}$, the integrand in (1.20) is smaller than a constant, $c_{3}$, times the the same integral but for $m_{\infty}$ replacing $m$. (By taking $T_{0}$ large, one can assume that $c_{3}<2$.) With the preceding understood, consider (1.20) with $m \equiv m_{\infty}$. Write

$$
e^{-2 t} \cdot d t=-2^{-1} \cdot d\left(e^{-2 t}\right)
$$

and integrate by parts in (1.20). Since $w$ is assumed to have compact support on $[T, \infty) \times S^{3}$, there will be no boundary contribution. The result is an equality between (1.20) and

$$
\int_{[T, \infty) \times S^{3}} e^{-2 t} \cdot\left\langle\partial_{t} w, w\right\rangle_{m} d t \cdot d \operatorname{vol}_{m} .
$$

Hölder's inequality applied to (1.22) finds (1.20) bounded by

$$
\int_{[T, \infty) \times S^{3}} e^{-2 t} \cdot\left|\partial_{t} w\right|_{m}^{2} d t \cdot d \operatorname{vol}_{m} .
$$

This 1st integral is written using the metric $m_{\infty}$, but with the addition of a constant $c_{4}$ in front, (1.23) with the metric $m$ is seen to bound (1.20) with the same metric $m$. (If $T_{0}$ is large, one can assume $c_{4}<2$.) 
Lemma $1.1^{\prime}$ follows now because consists (1.23) is no larger than $e^{-2 T}$ times (1.19).

\section{Proof of Theorem 2}

The proof in Section 2 for the unique continuation theorem can be modified to prove Theorem 2 of the Introduction. The details of this modification, and the resulting proof of Theorem 2 are given in this subsection.

To begin, note that it is sufficient, after Theorem 1, to consider the case where operatornameW $=M \times[0,1)$ and with the metric, $g$, any smooth Riemannian metric on $M \times[0,1)$. This will henceforth be assumed.

For the metric $g$ as above, the exponential map along geodesics normal to the boundary gives a $\delta>0$ and an embedding $\Psi: M \times[0, \delta) \rightarrow M \times[0,1)$ with

(1) $\Psi: M \times\{0\} \rightarrow M \times\{0\}$ is the identity.

(2) $\Psi^{*} g=d t^{2}+m$.

Here, $m$ is a smooth map from $[0, \delta)$ into the Frechet space of smooth metrics on $M$. With (2.1) understood, notation will be simplified by assuming that $\Psi$ is the identity map and that the metric $g$ has the a priori form $d t^{2}+m$ with $m$ a described above.

Now, suppose that $A$ and $A^{\prime}$ are a pair of $g$-anti self dual connections on the principal $G$-bundle $(M \times[0,1)) \times G$ which are gauge equivalent upon restriction on $M \times\{0\}$. To be precise, introduce $i: M \rightarrow M \times[0, \delta)$ to denote the boundary inclusion. By assumption, $\eta: M \rightarrow G$ should exist such that $i^{*} A^{\prime}=i^{*} A+\eta^{-1} \cdot d_{A} \eta$.

Use parallel transport to extend $\eta$ to a map from $M \times[0, \delta)$ to $G$ with the property that

$$
A^{\prime}=A+\eta^{-1} \cdot d_{A} \eta+a
$$

where $a$ is a $\mathfrak{g}$-valued 1 -form on $M \times[0, \delta)$ which obeys
(1) $\left.a\right|_{t=0}=0$,
(2) $\partial_{t} \iota a \equiv 0$. 
With (2.2) understood, remark that there is no generality lost vis à vis Theorem 2 to consider only the case $\eta \equiv 1$ in (2.2). (Change notation so that $A$ henceforth denoted (2.2)'s $A+\eta^{-1} \cdot d_{A} \eta$.)

Because $A$ and $A^{\prime}$ are assumed anti-self dual, the 1-form $a$ obeys

$$
P_{+}\left(d_{A} a+a \wedge a\right)=0
$$

where $P_{+}$is the self-dual projection for the metric $d t^{2}+m$. It follows from $(2.3,4)$ that there is a constant $c_{1}$ which is such that

$$
\left|P_{+} d_{A} a\right|_{g} \leq c_{1} \cdot t^{1 / 2} \cdot|a|_{g}
$$

holds at all times $t \in(0, \delta)$. In this form (with the factor $T^{1 / 2}$ instead of $t$ ), a Sobolev inequality shows that the constant $c_{1}$ depends only on the $L_{3}^{2}$ norm of $a$.

Lemma 2.1. There exists an increasing function $\tau:[0, \delta) \rightarrow[0, \infty)$ and a constant $c>0$ with the following properties: Let $t \in(0, \delta)$ and let $v$ be $a$ $\mathfrak{g}$-valued 1-form with compact support on $M \times[0, t)$ whose contraction with $\partial_{t}$ vanishes. Then, for all $\alpha \geq 0$, the following inequality holds:

$$
c \cdot t^{2} \int_{M \times[0, t)} e^{-2 \alpha \cdot \tau}\left|P_{+} d_{A} v\right|_{g}^{2} \cdot d \operatorname{vol}_{g} \geq \int_{M \times[0, t)}\left|e^{-2 \alpha \cdot \tau} \cdot v\right|_{g}^{2} \cdot d \operatorname{vol}_{g} .
$$

(Note that $\tau(t)=\int_{0}^{t} e^{-2 c_{2} s} d s$ for an appropriate constant $c_{2}>0$.)

Proof of Lemma 2.1. Introduce $\underline{d}_{A}$ to denote the covariant derivative along the fibers of the projection from $M \times[0, t)$ to $[0, t)$. Note that $(2.6)$ is true if $w \equiv e^{-\alpha \cdot \tau} \cdot v$ satisfies

$$
c \cdot t^{2} \int_{M \times[0, t)}\left|\partial_{t} w+\alpha \cdot f \cdot w+* \underline{d}_{A} w\right|_{m}^{2} \cdot d \operatorname{vol}_{g} \geq \int_{M \times[0, t)}|w|_{m}^{2} \cdot d \operatorname{vol}_{g}
$$

where $f=\partial_{t} \tau$.

consider first the integral on the left side of (2.7). It is equal to

$$
\int_{M \times[0, t)}\left(\left|\partial_{t} w\right|_{m}^{2}+\left|\alpha \cdot f \cdot w+* \underline{d}_{A} w\right|_{m}^{2}+2 \cdot\left\langle\partial_{t} w, \alpha \cdot f \cdot w+* \underline{d}_{A} w\right\rangle_{m}\right) \cdot d \operatorname{vol}_{g}
$$


The claim is that there is a choice of function $f$ (and hence, of $\tau$ in (2.6)) which makes each term in (2.8) positive whenever $W$ is a $\mathfrak{g}$-valued 1-form on $M \times[0,1)$ with no $d t$ component.

To prove this claim, note first that the integrand in (2.8) is observedly a sum of three terms, the first two of which are clearly non-negative. Integration by parts shows that the integral of the last term in the integrand of (2.8) is equal to

$$
\alpha \cdot \int_{M \times[0, t)}\left\langle w,-\partial_{t} f \cdot w+f \cdot H \cdot w\right\rangle_{m} \cdot d \operatorname{vol}_{g},
$$

where $H$ is a smooth map from $[0, t)$ into the vector space of smooth endomorphisms of $T^{*} M$. (Note that $H$ is computable from $\partial_{t} m$, and vanishes if the latter is zero.) In particular, there is a constant, $c_{2}>0$, which is such that

$$
\left|\langle\zeta, H \cdot \zeta\rangle_{m}\right| \leq c_{2} \cdot|\zeta|^{2}
$$

for any covector $\zeta$ and at any time $t \in[0, \delta)$.

Set $f \equiv e^{-2 c_{2} \cdot t}$, with $c_{2}$ as in (2.10). This choice of $f$ makes (2.9) nonnegative and completes the proof of the claim about (2.8).

The claim about (2.8) implies that the integral on the left side of (2.7) is at least as large as

$$
\int_{M \times[0, t)}\left|\partial_{t} w\right|_{m}^{2} d \operatorname{vol}_{g} .
$$

Now, the metric $m$ is everywhere close to the $t$-independent metric $m_{0} \equiv$ $\left.m\right|_{t=0}$ on $M \times[0, t)$ (if $\delta$ is small), and $w$ has compact support, so (2.11) is at least as large as

$$
\int_{M \times[0, t)}\left|\partial_{t} w\right|_{m}^{2} d \operatorname{vol}_{g} \geq c_{1} \cdot t^{-2} \int_{M \times[0, t)}|w|_{m}^{2} d \operatorname{vol}_{g} .
$$

This last equation proves (2.7).

To finally prove Theorem 2 , fix a non-negative function $\beta$, on $[0,1]$ which equals 1 on $[0,1 / 2]$, and equals 0 on $[3 / 4,1]$. Then, for $t<\delta$, introduce $\beta_{t}:[0, \delta) \rightarrow[0,1]$ whose value at $s$ is equal to $\beta(s / t)$. Set $v \equiv \beta_{t} \cdot a$ with $a$ 
as in $(2.3-5)$. Then $(2.5,6)$ imply that there exists $c_{3}>0$ such that for small $t>0$ and for all $\alpha>0$, one has

$$
c_{3} \cdot \int_{M \times[t / 2, t)} e^{-2 \alpha \cdot \tau} \cdot|v|_{m}^{2} d \operatorname{vol}_{g} \geq \int_{M \times[0, t / 2]} e^{-2 \alpha \cdot \tau} \cdot|v|_{m}^{2} d \operatorname{vol}_{g} .
$$

Since $\tau$ is increasing, (2.13) can hold for all $\alpha>0$ if and only if $a$ vanishes on some open neighborhood of $M \times\{0\}$ in $M \times[0,1)$. Thus, $A$ and $A^{\prime}$ are gauge equivalent on an open set, and then an application of Theorem 1 shows that $A$ and $A^{\prime}$ are locally gauge equivalent on all of $W$.

\section{Proof of Theorem 3}

The assertion that $\iota$ is $G$-equivarient follows by construction. The assertion that $\iota$ is smooth follows from a Sobolev embedding theorem, see [T]. The immersion assertion will follow if one can establish the following claim:

Proposition 3.1. Let $A$ be a smooth, anti-self dual connection on $\operatorname{int}(W)$ which is Sobolev class $L_{3}^{2}$ near $W$ 's boundary. Let a be a Sobolev class $L_{3}^{2}$ section over $W$ of $T^{*} W \otimes\left(P \times_{\mathrm{ad} G} \mathfrak{g}\right)$ which obeys $P_{+} d_{A} a=0$. Suppose that $i^{*} a=d_{A} \phi$ for some $L_{7 / 2}^{2}$ section $\phi$ over $M$ of $\left.P\right|_{M} \times_{\mathrm{ad} G} \mathfrak{g}$. Then every point in $W$ has a neighborhood on which a's restriction is $d_{A}$ (something), where "something" is a Sobolev class $L_{4}^{2}$ section over the neighborhood in question of the vector bundle $P \times_{\mathrm{ad} G} \mathfrak{g}$.

The immersion assertion of Theorem 3 follows from this proposition when the tangent space to $\mathfrak{M}^{0}$ at any orbit $[A]$ (of a connection $A$ on $P$ ) is written as the direct sum of two vector spaces. The first is the Lie algebra of the stabilizer of $[A]$ under the natural action of $S O(3)$ on $\mathfrak{M}^{0}$. The second is the vector space quotient $\operatorname{ker}\left(P_{+} d_{A}\right) / \operatorname{im}\left(d_{A}\right)$, where $\operatorname{ker}\left(P_{+} d_{A}\right)$ are the Sobolev class $L_{3}^{2}$ sections over $w$ of $T^{*} W \otimes\left(P \times_{\text {ad } G} \mathfrak{g}\right)$ which are annihilated by $P_{+} d_{A}$. With regard to Theorem 3 , the tangent space to $B^{0}(M)$ at an orbit, $[\Gamma]$ (of a connection $\Gamma$ ) should also be written as the direction sum of two vector spaces. The first is the Lie algebra of the stabilizer of $[\Gamma]$ under the action of $S O(3)$. The second is the quotient of the vector space $L_{5 / 2}^{2}\left(T^{*} M \otimes\left(\left.P\right|_{M} \times_{\mathrm{ad} G} \mathfrak{g}\right)\right)$ by the image of the operator $d_{\Gamma}$. 
By mimicking the proof of Theorem 2, it is straightforward to show that Proposition 3.1 holds when $W$ is a suitable neighborhood of $M \times\{0\}$ inside $M \times[0,1)$ with an arbitrary smooth metric. (The precise form of $w$ will depend on this metric.) The general case of Proposition 3.1 then follows from the following local form of Theorem 1:

Proposition 3.2. Let $d s^{2}$ be a smooth Riemannian metric on a ball $B \subset \mathbb{R}^{4}$ containing 0 . Let $A$ be a $\left(d s^{2}\right)$-anti-self dual connection on $B \times G$ and let $a$ be a smooth $\mathfrak{g}$-valued 1 -form over $B$ which obeys $P_{+} d_{A} a=0$. Let $\phi$ be a smooth $\mathfrak{g}$-valued function on $B$ such that $a-d_{A} \phi$ vanishes to infinite order at 0 . Then $a=d_{A} \phi^{\prime}$, where $\phi^{\prime}$ is a smooth, $\mathfrak{g}$-valued function $B$.

Proof of Proposition 3.2. Mimic the proof of Theorem 1. To be precise, the given assumption imply that there exists a $\mathfrak{g}$-valued function $\phi_{1}$ on $B$ such that $u \equiv a-d_{A} \phi_{1}$ vanishes to infinite order at 0 and also annihilates any vector on $B$ which is tangent to a radial geodesic through 0 . Fix $r>0$, and apply Lemma 1.1 to $v-a_{2} \equiv \beta_{r} \cdot u$, where $\beta_{r}$ is as described in Section 1 . Therefore, if $r$ is small, then $u$ will obey (1.7) for all $\alpha>0$, thus proving that $u \equiv 0$ on some ball about 0 .

\section{Proof of Theorem 4}

To prove Theorem 4, introduce a set $U \subset W$ which is defined as follows: A point $p \in U$ if there is a section of $P \times_{G} \mathfrak{g}$ which is non-vanishing at $p$ and which is $A$-covariantly constant on a neighborhood of $p$. This set is open, by definition. Theorem 4 is proved by establishing that $U$ is non-empty and also closed. The proof that $U$ is non-empty also establishes that $U$ is closed. The argument goes as follows: Let $p$ be a point where $A$ is reducible to infinite order. Trivialize $P$ near $p$ so that $A=\Gamma+a$, where $\Gamma$ is the product connection and where $a$ is a $\mathfrak{g}$-valued 1-form which vanishes at $p$ and which annihilates any tangent vector to any radial geodesic through $p$. Suppose that $\phi$ is a $\mathfrak{g}$-valued function near $p$ which is non-zero near $p$ and which is such that $d_{A} \phi$ vanishes to infinite order near $p$. Let $\sigma \equiv \phi(p) \in \mathfrak{g}$. For small, nonzero $\epsilon$, introduce $\eta \equiv \exp (\epsilon \cdot \sigma) \in G$. The connections $\Gamma+a$ and $\Gamma+\eta^{-1} a \eta$ are both anti-self dual near $p$, and they agree to infinite order at $p$. The proof of Theorem 1 
shows that they are equal on a neighborhood of $p$. Since this is true for all $\epsilon$, the constant $\mathfrak{g}$-valued function which sends the neighborhood of $p$ to $\sigma$ is $A$-covariantly constant.

\section{Proof of Theorem 5}

Suppose that $i^{*} A$ is $\underline{G}$-reducible on $M$. The proof of Theorem 5 has two parts. Part 1 establishes that $i^{*} A$ is $\underline{G}$-reducible on some neighborhood of $M \times\{0\} \subset M \times[0,1) \subset W$. Part 2 establishes that the set of points in $W$ which have neighborhoods where $A$ is $\underline{G}$-reducible is open and closed. Since this set is non-empty, it will equal $W$.

To prove the Part 1, use geodesic normal coordinates to give a diffeomorphism from a neighborhood of $M \times\{0\}$ to $M \times[0, \delta)$ for some $\delta>0$. Let $\pi: M \times[0, \delta) \rightarrow M$ denote the resulting projection.

Parallel transport by $A$ along the fibers of $\pi$ defines a bundle isomorphism

$$
\phi:\left.\left.P\right|_{M \times[0, \delta)} \rightarrow \pi^{*} P\right|_{M} .
$$

This isomorphism has the property that $A=\phi^{*} i^{*} A+a$, where $a$ vanishes on $\{0\} \times M$ and annihilates any tangent vector to any fiber of $\pi$.

Use $\rho$ to define $A$ as a connection on $P \times{ }_{\rho} \underline{G}$. The hypothesis that $A$ is reducible along $M$ means that there is a $i^{*} A$-covariantly constant section, $h$, of $\left.P\right|_{M} \times{ }_{\mathrm{ad}} \rho \underline{G}$. As connections on $P \times{ }_{\rho} \underline{G}$ over $M \times[0, \delta)$, both $\phi^{*} i^{*} A+a$ and also $\phi^{*} i^{*} A+h \cdot a \cdot h^{-1}$ are anti-self dual. They agree on a neighborhood of $M \times\{0\}$. The proof of Theorem 2 shows that they agree on a neighborhood of $M \times\{0\}$. Thus, $h$ is $A$-covariantly constant on a neighborhood of $M \times\{0\}$ in $W$. (Given that $h$ is $i^{*} A$-covariantly constant, the assertion that the two connections agree and the assertion that $h$ is $A$-covariantly constant are two ways to say the same thing.)

The proof of Part 2 mimics the proof of Proposition 3.2 and it is left to the reader.

\section{REFERENCES}

[Ar] Aronszajn, N., A unique continuation theorem for solutions of elliptic partial differential equations of the second order, J. Math. Pure Appl. 36 (1957), 235-249. 
[AHS] Atiyah, M. F., Hitchin, N. J. and Singer, I. M., Self duality in 4-dimensional Riemannian geometry, Proc. Roy. Soc. Lond. A 308 (1982), 523-615.

[Ad] Adams, R., Sobolev Spaces, Academic Press, New York 1975.

[CJS] Cohen, R., Jones, J. D. S. and Segal, G., Floer's infinite dimensional Morse theory and homotopy theory, in the Andreas Floer Memorial Volume, ed. by C. H. Taubes, A.Weinstein and E. Zehnder, Springer-Verlag, to appear.

[D] Donaldson, S. K., Boundary value problems for Yang-Mills fields, preprint 1992.

[DK] Donaldson, S. K. and Kronheimer, P. B., The Geometry of 4-manifolds, Oxford University Press, 1990.

[F] Floer, A., An instanton invariant for 3-manifolds, Comm. Math. Phys. 118 (1988), 215-240.

[M] Marini, A., Dirichlet and Neumann boundary value problems for Yang-Mills connections, Comm. Pure Appl. Math. XLV (1992), 1017-1050.

[T] Taubes, C. H., $L^{2}$-moduli spaces on 4-manifolds with cylindrical ends, International Press, Boston, to appear.

[TWZ] Taubes, C. H., Weinstein, A. and Zehnder E. eds. for the Andreas Floer Memorial Volume, Springer-Verlag, to appear.

[U] Uhlenbeck, K. K., Connections with $L^{2}$ bounds on curvature, Commun. Math. Phys. 83 (1982), 31-42.

HARVARD UNIVERSity, U. S. A.

RECEIVEd June 7, 1993. 